\title{
Evolution from $A+2$ Defect to +1/2 Defects in a Cylindrical Geometry
}

\author{
Dmitri Miroshnychenko \\ N. A. Hill \\ Department of Mathematics, University of Glasgow, University \\ Gardens, Glasgow, United Kingdom
}

\section{N. J. Mottram}

Department of Mathematics, University of Strathclyde, Livingstone

Tower, Glasgow, United Kingdom

\section{J. E. Lydon}

School of Biochemistry and Microbiology, University of Leeds, Leeds, United Kingdom

In this work the dynamics of liquid crystal ordering in a cylindrical geometry are considered. We study a system with liquid crystalline properties that exhibits translational symmetry along the cylinder axis and, therefore, the problem is effectively two-dimensional. The orientation of liquid crystals is described by a tensorial order parameter and the dynamics are governed by a balance between the dissipation and the rate of change of free energy, which includes the elastic, thermotropic and surface energy terms. The evolution of the +2 defect differentiating first into two +1 disclinations and subsequently into four $+1 / 2$ defects is analysed. Different boundary conditions, namely strong and weak or no anchoring, have been considered and the critical value for the anchoring strength, at which $+1 / 2$ defects are very close to escaping through the boundary but still remain there at equilibrium, has been identified.

Keywords: anchoring; dislocation dynamics; dissipation; free energy; ordering; structures

Address correspondence to Dmitri Miroshnychenko, Department of Mathematics, University of Glasgow, University Gardens, Glasgow G12 8QW, United Kingdom. E-mail: dm@maths.gla.ac.uk 


\section{INTRODUCTION}

Existing, new and emerging electro-optic technologies have focused the efforts of many engineers, physicists and chemists on the applications of liquid crystals in flat-panel displays. It is the susceptibility of liquid crystals to external (electric or magnetic) fields [1-3] combined with the discovery of polymer-dispersed liquid crystal materials [4-6] that suggested the applications of spherical liquid crystal droplets in display technologies. A great deal of current studies on liquid crystals are now centred around this theme and a good historical overview including the avenues of present research in that area can be found in [7].

Commercial use of liquid crystals is not the only interest for scientists and academic curiosity in these ubiquitous, versatile materials led them to explore other areas of research as well. For instance, some biological tissues have liquid crystal structures and many biologists, medical researchers and pharmacists are now also studying and exploiting liquid crystals. Studies into the astonishing properties (for example, growth [8]) of liquid crystals can even provide an understanding into the origin of life.

Rich and intriguing physical phenomena of ordering and defects in liquid crystals confined to curved geometries also attracted considerable attention. Systems with simple cylindrical symmetry were employed to study the elastic properties of liquid crystals. Details on various aspects of dislocation theory can be found in [9].

Different nematic director fields of liquid crystals constrained to cylindrical environments have been studied [10-14]. Homeotropic (perpendicular) boundary conditions were applied on the cylindrical walls. The escaped radial-director-field configuration, possibly forming a point defect on the cylinder axis, was found to be of lower energy than the planar radial-director-field predicted earlier. Defects occur often in liquid crystals and they can be of different strength [15]; however, much of the attention has been given to nematics with a +1 type of disclination.

In this work, the dynamics of liquid crystal ordering with the initial director configuration prescribed by a +2 planar defect placed in the centre of a cross-section of a circular cylinder are considered. We study the model system with liquid crystalline properties that exhibits translational symmetry along the central axis of the cylinder and, therefore, the problem is effectively reduced to two dimensions. And though we have not constrained the system, no escape into the third dimension, along the cylinder axis, takes place due to the relatively small length scale (radius) and possibly due to the high speed of defect separation compared to escape. 
The liquid crystal orientation is described by a second rank tensor order parameter and the dynamics are governed by a balance between the dissipation and the rate of change of free energy. The total free energy considered includes the elastic energy of distortion of the structure, the thermotropic energy that dictates a preferred phase, and the surface energy representing the interaction between the bounding surface and adjacent liquid crystal molecules. To solve the governing equations, a programme using a finite element method has been employed.

First we look at the short time behaviour of the unstable +2 defect. The decay occurs in a cascade going from two plus; 1 disclinations to four $+1 / 2$ defects. The mechanism underlying the dynamics of this disclination topology is discussed. Secondly, after the final division into four $+1 / 2$ disclinations, the defects either travel to their equilibrium positions and stay there, or they escape through the boundary. Their destination will be dictated by the nature and strength of anchoring the nematic at the bounding surface.

\section{TENSOR ORDER PARAMETER}

The concept of an order parameter defining the amount of orientational order is not new and details on the scalar or tensor order parameter can be found in [17]. The tensor order parameter is used to remove problems [18] with the degeneracies of the Euler angles and to be able to investigate the dynamics of defects. For that purpose, a symmetric traceless tensor of second rank

$$
\mathbf{Q}=S_{1}\left(\mathbf{n} \otimes \mathbf{n}-\frac{1}{3} \mathbf{I}\right)+S_{2}\left(\mathbf{m} \otimes \mathbf{m}-\frac{1}{3} \mathbf{I}\right)
$$

is introduced, where $S_{1}$ and $S_{2}$ are two scalar order parameters associated with order about two directors $\mathbf{n}$ and $\mathbf{m}$ respectively, and $\mathbf{I}$ is the identity tensor.

The use of a traceless tensor means that the isotropic state is represented by $\mathbf{Q}=\mathbf{0}$, which makes the expansion of the free energy about the isotropic state relatively simple. The directors $\mathbf{n}$ and $\mathbf{m}$ are two perpendicular vectors (the third direction of symmetry is determined automatically from these two as $\mathbf{n} \times \mathbf{m}$ ) that fully define the orientation of a liquid crystal in the most general nematic state that corresponds to biaxial phase. Note also that the form of the tensor order parameter (1) reflects the nematic symmetry, i.e., the physical equivalence of $\mathbf{n}$ and $-\mathbf{n}$ as well as of $\mathbf{m}$ and $-\mathbf{m}$. 
Both vectors $\mathbf{n}$ and $\mathbf{m}$ are of unit length and they can be described (Fig. 1) in terms of the Euler angles $\varphi, \theta, \psi$ as follows:

$$
\begin{aligned}
\mathbf{n}= & (\cos \varphi \cos \theta, \sin \varphi \cos \theta, \sin \theta), \\
\mathbf{m}= & (\sin \varphi \cos \psi-\cos \varphi \sin \theta \sin \psi, \\
& -\sin \varphi \sin \theta \sin \psi-\cos \varphi \cos \psi, \cos \theta \sin \psi) .
\end{aligned}
$$

The director $\mathbf{n}$ is determined by the two angles $\varphi$ and $\theta$, while the director $\mathbf{m}$ needs only the additional variable, the Euler angle $\psi$, to describe it as well. $\psi$ is measured from the direction in the $x_{1} x_{2}$-plane, $(\sin \varphi,-\cos \varphi, 0)$, that is also perpendicular to $\mathbf{n}$. Here the Euler angles $\varphi, \theta, \psi$ have their standard meaning with respect to laboratory frame of reference with axes $x_{1}, x_{2}, x_{3}$.

In fact, both vector variables $\mathbf{n}(\mathbf{x}, t)$ and $\mathbf{m}(\mathbf{x}, t)$ and scalar variables $S_{1}(\mathbf{x}, t)$ and $S_{2}(\mathbf{x}, t)$ depend on the spatial coordinates $\mathbf{x}$ and time $t$. Thus, there are only five separate dependent variables

$$
\varphi(\mathbf{x}, t), \theta(\mathbf{x}, t), \psi(\mathbf{x}, t), S_{1}(\mathbf{x}, t), S_{2}(\mathbf{x}, t),
$$

and that fact is reflected in the tensor order parameter (1) as well; it has also five independent components. It is convenient to rewrite the traceless tensor $\mathbf{Q}$ in the form

$$
\mathbf{Q}=\left(\begin{array}{ccc}
q_{1} & q_{2} & q_{3} \\
q_{2} & q_{4} & q_{5} \\
q_{3} & q_{5} & -q_{1}-q_{4}
\end{array}\right)
$$

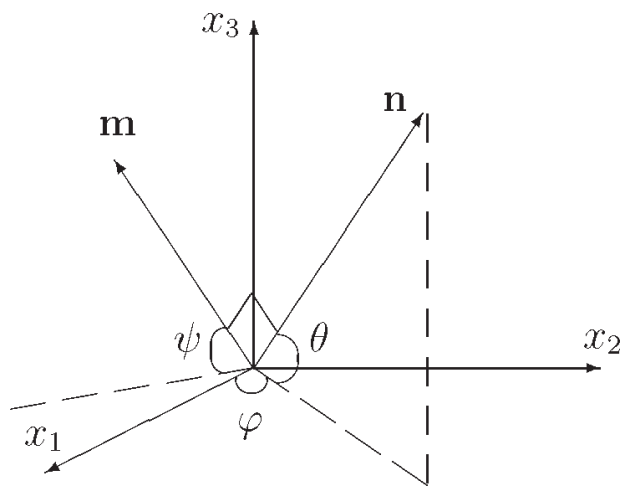

FIGURE 1 Surface plot for the scalar order parameter $S_{1}$. The directors $\mathbf{n}$ and $\mathbf{m}$ in terms of the Euler angles $\varphi, \theta, \psi$. The axes $x_{1}, x_{2}, x_{3}$ from the laboratory frame of reference. 
where

$$
\begin{aligned}
q_{1}= & S_{1} \cos ^{2} \theta \cos ^{2} \varphi+S_{2}(\sin \varphi \cos \psi+\cos \varphi \sin \psi \sin \theta)^{2}-\frac{1}{3}\left(S_{1}+S_{2}\right), \\
q_{2}= & S_{1} \cos ^{2} \theta \sin \varphi \cos \varphi \\
& -S_{2}(\cos \varphi \cos \psi-\sin \varphi \sin \psi \sin \theta)(\sin \varphi \cos \psi+\cos \varphi \sin \psi \sin \theta), \\
q_{3}= & S_{1} \sin \theta \cos \theta \cos \varphi-S_{2} \sin \psi \cos \theta(\sin \varphi \cos \psi+\cos \varphi \sin \psi \sin \theta), \\
q_{4}= & S_{1} \cos ^{2} \theta \sin ^{2} \varphi+S_{2}(\cos \varphi \cos \psi-\sin \varphi \sin \psi \sin \theta)^{2}-\frac{1}{3}\left(S_{1}+S_{2}\right), \\
q_{5}= & S_{1} \sin \theta \cos \theta \sin \varphi+S_{2} \sin \psi \cos \theta(\cos \varphi \cos \psi-\sin \varphi \sin \psi \sin \theta) .
\end{aligned}
$$

Restating the problem in terms of five variables $q_{i}(i=1 \ldots 5)$ avoids difficulties due to the multivaluedness of the Euler angles, and the use of the components $q_{i}$ removes the degeneracy when $\theta=\pi / 2$.

\section{DISSIPATION AND FREE ENERGY}

The dynamical equations for a nematic liquid crystal with the tensor order parameter can be determined from a variational principle originally put forward by Rayleigh [19]. In a conservative system this principle asserts that the dissipation of energy evolves at a minimum rate relative to all virtual values it could develop due to variations $\delta \dot{q}_{i}$. That requirement results [20] in the balance of energy variations given as

$$
\delta \mathscr{R}+\delta \dot{\mathscr{F}}=0,
$$

in which $\mathscr{R}$ is the Rayleigh dissipation function and $\dot{\mathscr{F}}$ is the rate of change of free energy.

It is assumed that the rate of dissipation, $R$, is a functional invariant of $\dot{\mathbf{Q}}$ and, therefore, it can be written approximately in the positive-definite quadratic form leading to

$$
\mathscr{R}=\int_{V} R d V=\int_{V} \gamma \operatorname{tr} \dot{\mathbf{Q}}^{2} d V
$$

where $\gamma$ is related to the rotational viscosity of the nematic liquid crystal and $V$ is a volume in space. The variation of the dissipation function can be determined easily from the expression (6), and its density reads

$$
\frac{\partial R}{\partial \dot{q}_{i}} \delta \dot{q}_{i}=2 \gamma \operatorname{tr}\left(\dot{\mathbf{Q}} \frac{\partial \mathbf{Q}}{\partial q_{i}}\right) \delta \dot{q}_{i},
$$

where the summation over repeated indices is used. 
The free energy considered here includes the elastic energy of distortion of the structure, the thermotropic energy that dictates a preferred (isotropic or nematic) state, and the surface energy representing the interaction between the bounding surface and adjacent liquid crystal molecules. Thus,

$$
\mathscr{F}=\int_{V}\left(F_{\mathrm{d}}+F_{\mathrm{t}}\right) d V+\int_{S} F_{\mathrm{s}} d S,
$$

where the energy densities $F_{\mathrm{d}}, F_{\mathrm{t}}, F_{\mathrm{s}}$ correspond to the terms named above respectively, $S$ is the bounding surface of the volume $V$. It is assumed further that the densities for the surface energy and for the thermotropic energy are functions of the elements of $\mathbf{Q}$ only, so that

$$
F_{\mathrm{s}}=F_{\mathrm{s}}\left(q_{i}\right), \quad F_{\mathrm{t}}=F_{\mathrm{t}}\left(q_{i}\right),
$$

whereas the elastic (or distortional) energy density is also dependent on all first order spatial derivatives of $\mathbf{Q}$, i.e.,

$$
F_{\mathrm{d}}=F_{\mathrm{d}}\left(q_{i}, \nabla q_{i}\right) .
$$

Higher order differentials of $\mathbf{Q}$ are neglected here as the distortions of $\mathbf{Q}$ are regarded as small.

The density of the variation of free-energy rate-of-change may be given using the Euler-Lagrange theorem as

$$
\left[\frac{\partial F_{\mathrm{t}}}{\partial q_{i}}+\frac{\partial F_{\mathrm{d}}}{\partial q_{i}}-\frac{\partial}{\partial x_{j}}\left(\frac{\partial F_{\mathrm{d}}}{\partial q_{i, j}}\right)\right] \delta \dot{q}_{i}
$$

where a comma denotes differentiation with respect to a spatial coordinate. Since variations $\delta \dot{q}_{i}$ are arbitrary and $V$ may be any chosen volume, then relations (5), (7) and (9) can be restated in the form

$$
\frac{\partial R}{\partial \dot{q}_{i}}+\frac{\partial F_{\mathrm{t}}}{\partial q_{i}}+\frac{\partial F_{\mathrm{d}}}{\partial q_{i}}-\frac{\partial}{\partial x_{j}}\left(\frac{\partial \boldsymbol{F}_{\mathrm{d}}}{\partial q_{i, j}}\right)=0 \quad(i=1 \ldots 5),
$$

and these constitute the governing equations for the components of the tensor order parameter at every point of space. The system (10) is complemented by boundary conditions on the bounding substrate of the liquid crystal:

$$
\frac{\partial \boldsymbol{F}_{\mathrm{s}}}{\partial q_{i}}+\nu_{j} \frac{\partial \boldsymbol{F}_{\mathrm{d}}}{\partial q_{i, j}}=0 \quad(i=1 \ldots 5),
$$

where $v$ is the outward normal to the substrate.

The form of thermotropic energy density is taken [21] as an expansion in a Taylor series about $\mathbf{Q}=\mathbf{0}$ and only the first five 
terms (note that $\operatorname{tr} \mathbf{Q}=0$ ) are included which are sufficient to enable the isotropic and nematic states to be simultaneously stable. Thus,

$$
F_{\mathrm{t}}=F_{0}+a \operatorname{tr} \mathbf{Q}^{2}+\frac{2 b}{3} \operatorname{tr} \mathbf{Q}^{3}+c \operatorname{tr} \mathbf{Q}^{4},
$$

where $F_{0}$ is the energy density of the isotropic state and the coefficients $a, b, c$ are in general temperature dependent. It is, however, usual to approximate these coefficients by assuming that $b$ and $c$ are independent of temperature whilst $a=\alpha\left(T-T^{*}\right)$ where $\alpha>0$ and $T^{*}$ is the fixed temperature at which the isotropic state becomes unstable.

The elastic or distortional energy density of a liquid crystal is derived from the energy induced by distorting $\mathbf{Q}$ in space. As noted earlier, the elastic energy density depends on all spatial first-order derivatives of $\mathbf{Q}$. However, frame indifference and invariance over rotation or translation mean that not all combinations of $\mathbf{Q}$ derivatives are allowed. Taking that into account, the elastic energy density may

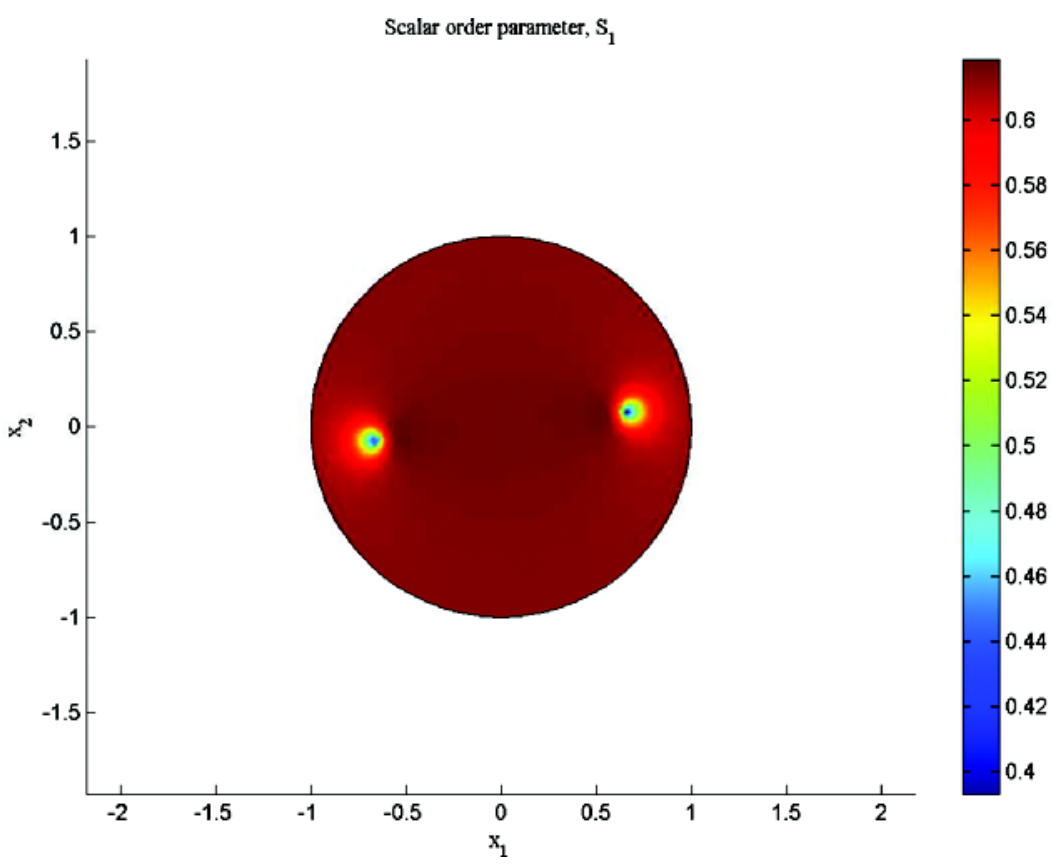

FIGURE 2 Surface plot for the scalar order parameter $S_{1}$. Equilibrium positions of two $+1 / 2$ defects are reached. $\bar{W} \gg 1$. 


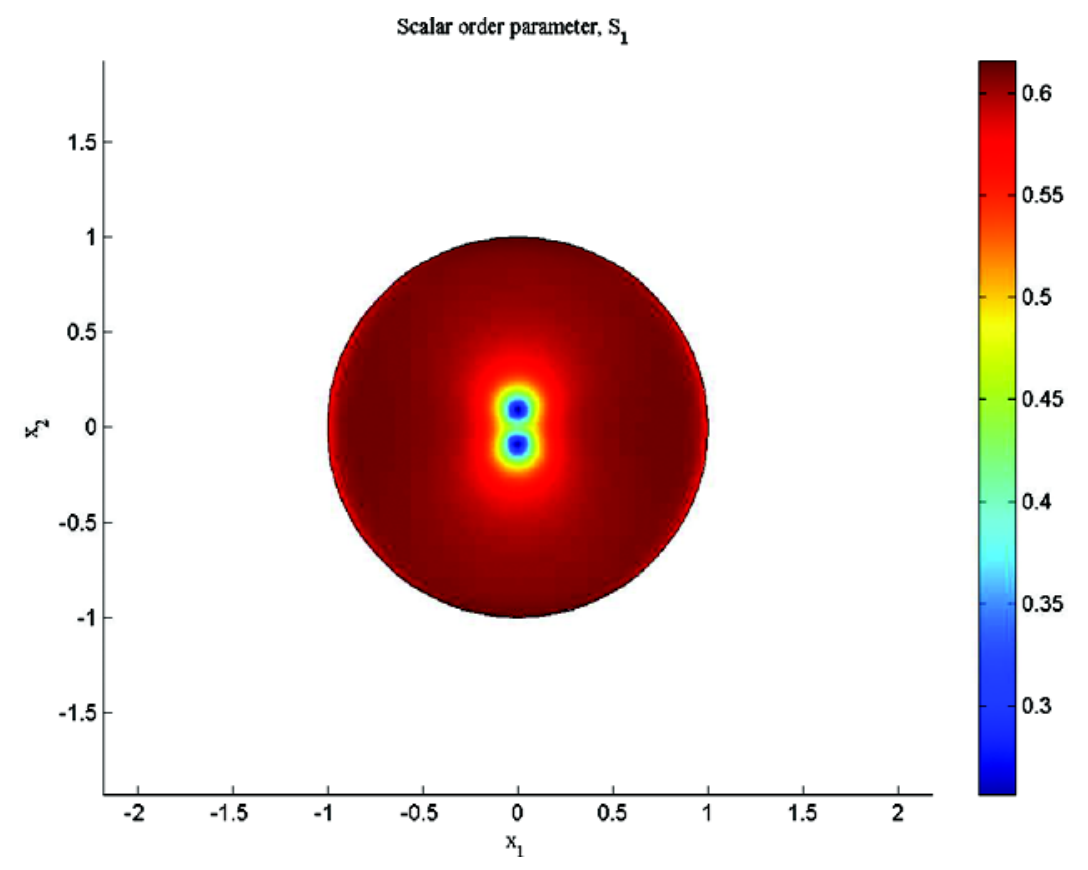

FIGURE 3 Surface plot for the scalar order parameter $S_{1}$. This shows the two +1 defect structure at $\bar{t}=0.001$. $W=0 \mathrm{~N} / \mathrm{m}$.

be expressed [23] as

$$
\begin{aligned}
\boldsymbol{F}_{\mathrm{d}}= & \frac{L_{1}}{2} \frac{\partial \boldsymbol{Q}_{i j}}{\partial x_{k}} \frac{\partial \boldsymbol{Q}_{i j}}{\partial x_{k}}+\frac{L_{2}}{2} \frac{\partial Q_{i j}}{\partial x_{j}} \frac{\partial Q_{i k}}{\partial x_{k}}+\frac{L_{3}}{2} \frac{\partial Q_{i k}}{\partial x_{j}} \frac{\partial \boldsymbol{Q}_{i j}}{\partial x_{k}} \\
& +\frac{L_{4}}{2} \epsilon_{l i k} \boldsymbol{Q}_{l j} \frac{\partial \boldsymbol{Q}_{i j}}{\partial x_{k}}+\frac{L_{6}}{2} \boldsymbol{Q}_{l k}+\frac{\partial \boldsymbol{Q}_{i j}}{\partial x_{l}} \frac{\partial \boldsymbol{Q}_{i j}}{\partial x_{k}},
\end{aligned}
$$

where the elastic parameters $L_{1}, L_{2}, L_{3}, L_{4}, L_{6}$ can be related [23] to the Frank elastic constants $k_{11}, k_{22}, k_{33}, k_{24}, q_{0}$, and $\epsilon_{l i k}$ is the LeviCivita alternating tensor. For a non-chiral liquid crystal $L_{4}=0$ as is the case here. The term with the coefficient $L_{6}$ ensures the mapping from the $\mathbf{Q}$ tensor to the Frank energy and allows $k_{11} \neq k_{33}$. However, we assume that $L_{6}=0$ and $k_{11}=k_{33}$ (and this is more accurate close to the nematic-isotropic transition temperature). Note that there are other elastic terms that are of cubic order in $\mathbf{Q}$ which could be included, but the equations become then extremely cumbersome and only the $L_{6}$ term in (13) is needed in order to break the $k_{11}=k_{33}$ degeneracy. 


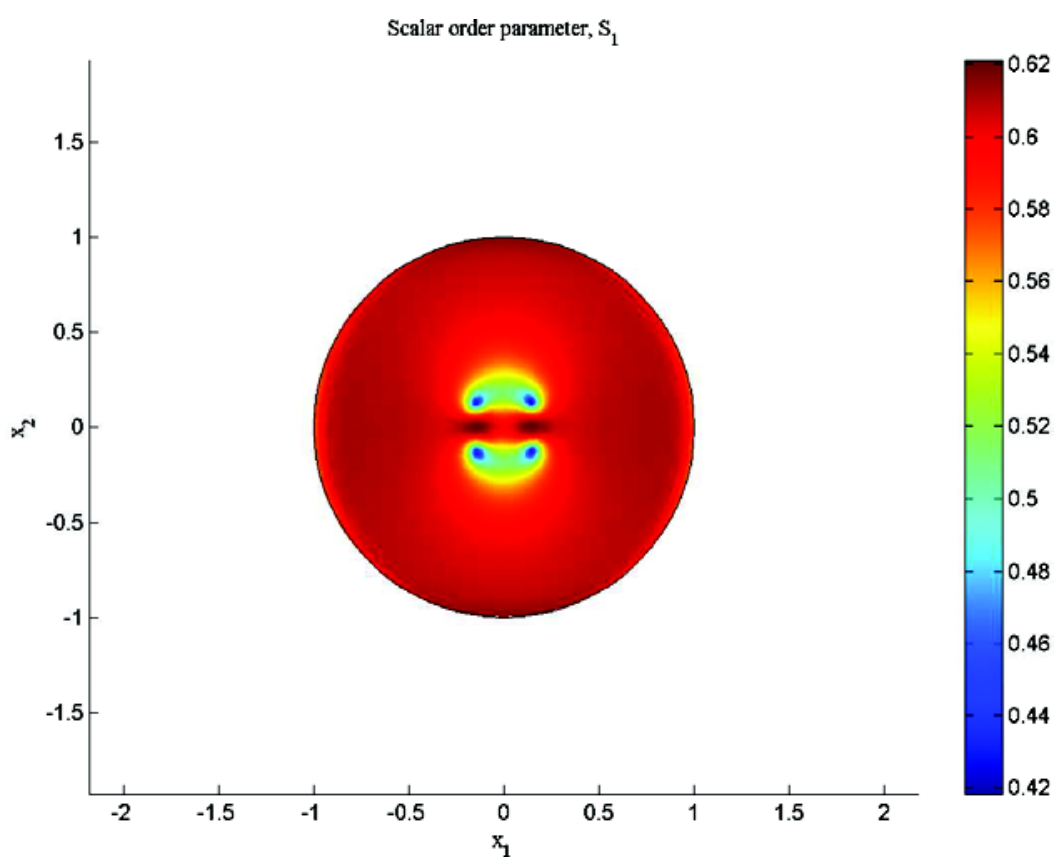

FIGURE 4 Surface plot for the scalar order parameter $S_{1}$. The four $+1 / 2$ defect structure at $\bar{t}=0.0056$ is shown. $W=0 \mathrm{~N} / \mathrm{m}$.

The form of surface energy density is determined by

$$
F_{\mathrm{s}}=\frac{W}{2} \operatorname{tr}\left(\mathbf{Q}-\mathbf{Q}_{\mathrm{s}}\right)^{2}
$$

where $\mathbf{Q}_{\mathrm{S}}$ is the value of the tensor order parameter preferred by the surface and $W$ is the anchoring strength. The density $F_{\text {s }}$ plays an important role in the competition between the surface energy and the bulk energy and it enters the dynamics through the boundary conditions (11).

\section{RESULTS AND DISCUSSION}

To solve numerically the governing equations (10), (11), we use the FEMLAB 3 programme, which uses a finite element method. Typical coefficients for the expansion (12) have been assigned based on the data in [22] for $5 \mathrm{CB}$, which is one of the very few papers to give physical values for these parameters: $\alpha=0.13 \times 10^{6} \mathrm{~N} / \mathrm{Km}^{2},\left(T-T^{*}=\right.$ $-5 \mathrm{~K}$ and, hence, $\left.a=-0.65 \times 10^{6} \mathrm{~N} / \mathrm{m}^{2}\right), b=-1.6 \times 10^{6} \mathrm{~N} / \mathrm{m}^{2}, c=$ 


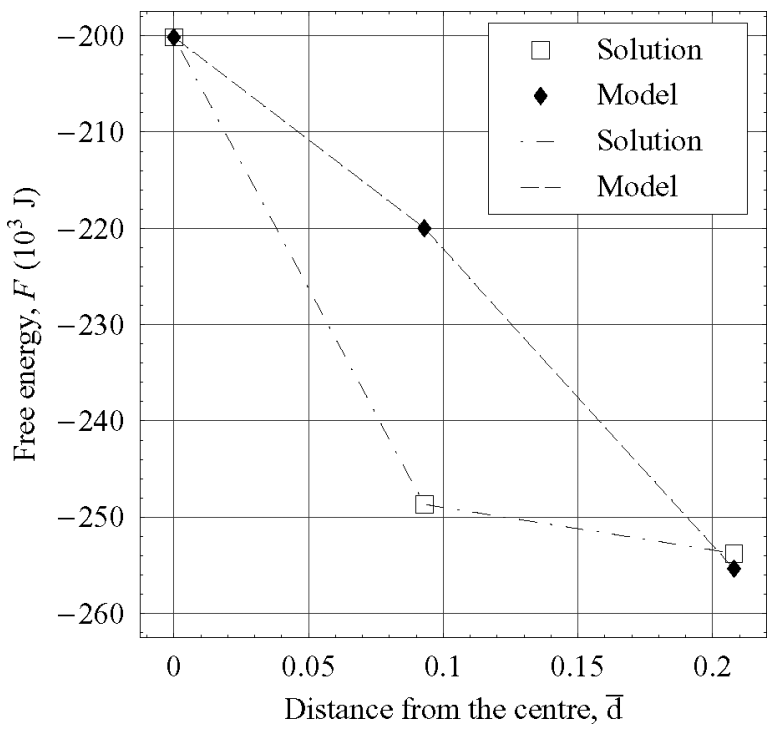

FIGURE 5 The free energy $\mathscr{F}$ computed $\left(F_{0}=0 \mathrm{~J}, W=0 \mathrm{~N} / \mathrm{m}\right)$ for three structures increasing with the distance $\bar{d}$ from the centre, these are: a +2 disclination at the centre, two +1 defects (at time $\bar{t}=0.001$ ), and subsequently four $+1 / 2$ defects $(\bar{t}=0.0056)$. Also shown is the free energy $\mathscr{F}$ calculated for model director fields with four $+1 / 2$ defects seeded at the same distance $\bar{d}$ from the centre as the corresponding structures in full numerical simulation. This shows that as the full numerical simulation evolves the free energy associated with the two +1 defects is lower than that of the four $+1 / 2$ defects at the intermediate time $\bar{t}=0.001$ when $\bar{d}=0.09$.

$3.9 \times 10^{6} \mathrm{~N} / \mathrm{m}^{2}$. The elastic constants have been chosen for $5 \mathrm{CB}$ from [23-27] and converted to: $L_{1}=0.92 \times 10^{-12} \mathrm{~N}, L_{2}=3.68 \times 10^{-12} \mathrm{~N}$, $L_{3}=0 \mathrm{~N}$. The initial condition is set to $\tan 2 \varphi=x_{2} / x_{1}$ (for a +2 defect), $\theta=0, \psi=0, S_{1}=0.613, S_{2}=0$. Although this is not an exact solution, it has the right topology to initiate a +2 defect. The radius of the cylinder is $r=0.0316 \mu \mathrm{m}$; spatial coordinates, time, and the anchoring strength are non-dimensionalised as $\bar{x}_{i}=x_{i} / r, \bar{t}=t \times 10^{3} / 2 \gamma$, and $\bar{W}=W r / L_{2}$ respectively; and $\gamma$ is usually of the order of $0.1 \mathrm{~Pa}$ s. All figures show the surface plots for the scalar order parameter $S_{1}$ apart from Figure 7 which shows $S_{2}$.

Different initial configurations for the director field - defects of $+1 / 2,+1,+3 / 2$ and +2 type placed in the centre of the circular cross-section of the cylinder - have been analysed. The preferred alignment on the substrate is homeotropic $\left(\tan \varphi=x_{2} / x_{1}, \theta=0\right.$, $\left.\psi=0, S_{1}=0.613, S_{2}=0\right)$ throughout. When strong anchoring 


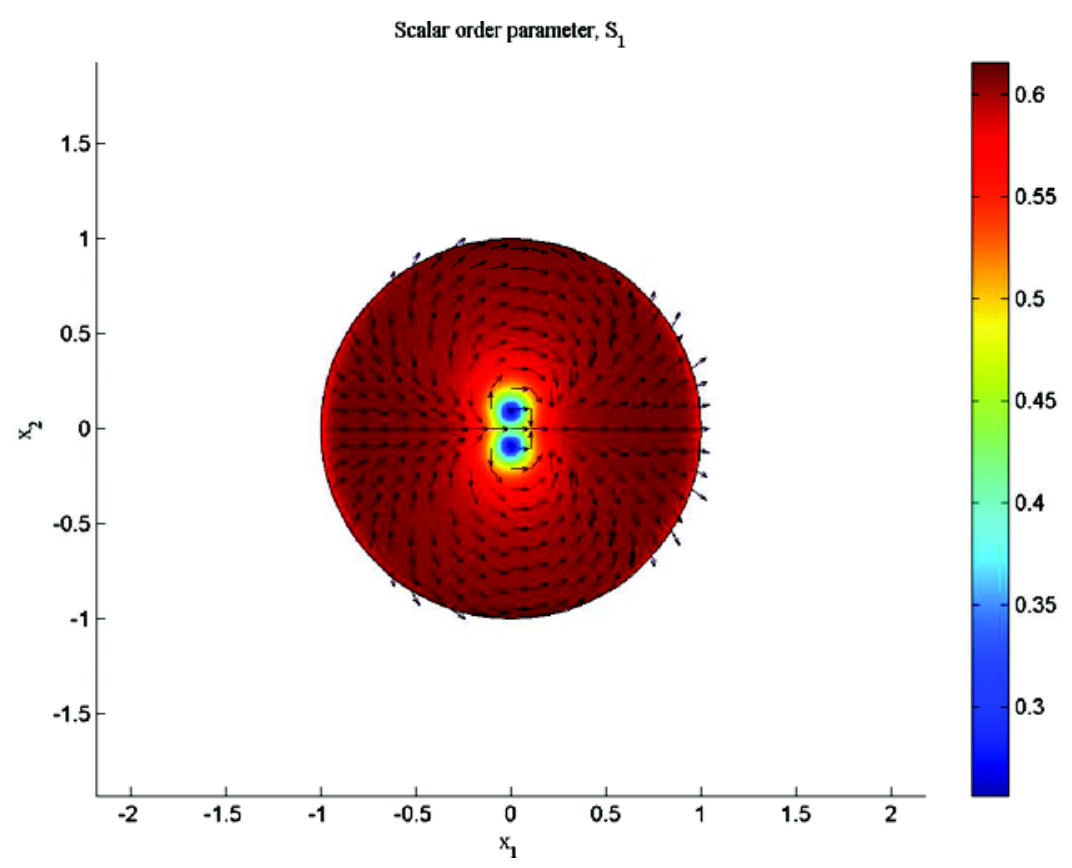

FIGURE 6 Surface plot for the scalar order parameter $S_{1}$. This shows the director field $\mathbf{n}$ for two +1 defect structure at $\bar{t}=0.001$. $W=0 \mathrm{~N} / \mathrm{m}$.

$(\bar{W} \gg 1)$ is applied, then the persistent leitmotif of the dynamics is two $+1 / 2$ disclinations repelling each other into equilibrium positions (Fig. 2 ) in a final static pattern.

This behaviour is determined by the homeotropic strong-anchoring boundary condition that strives to conserve the topological defect of +1 strength within the domain. Although the escape of a +1 defect into the third dimension is not disallowed, it does not take place (so that $\theta \equiv 0)$, as might be expected along the cylinder axis [10-14], due to the relatively small radius $(r \ll 1 \mu \mathrm{m})$ of the cylinder and possibly due to the high speed of defect separation compared to the escape process. It is the observed division of defects that leads to a very interesting behaviour in this system in general and with the +2 initial defect in particular.

\section{Defect Division}

From our computational results, we observe that the +2 disclination is a high-energy, unstable structure, which reorganises into four 


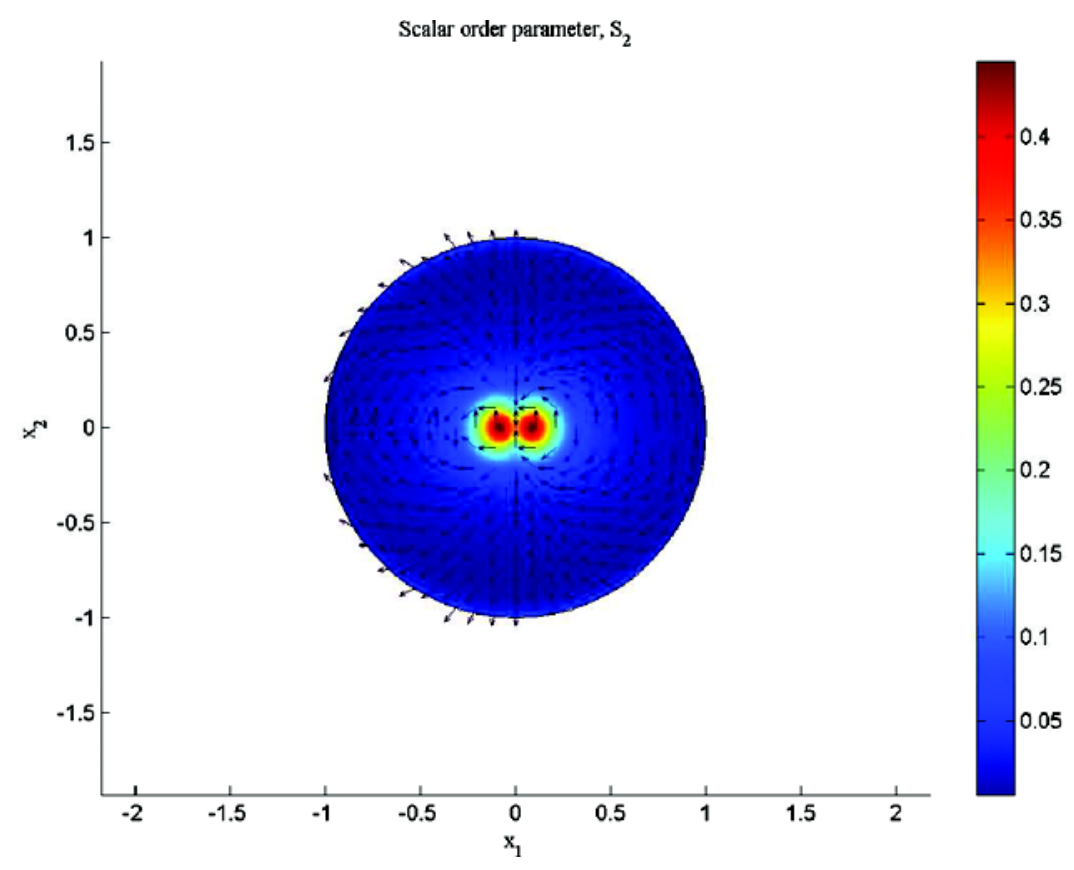

FIGURE 7 Surface plot for the scalar order parameter $S_{2}$. This shows the director field $\mathbf{m}$ for two +1 defect structure at $\bar{t}=0.001$. $W=0 \mathrm{~N} / \mathrm{m}$.

$+1 / 2$ defects. There exists, however, an intermediate stage (for any value of $W$ ) when the +2 defect undergoes a rapid differentiation into two +1 defects (Fig. 3), and only thereafter does the subsequent division into $+1 / 2$ defects take place (Fig. 4). From the Frank energy description the energies of a single +2 , two +1 and four $+1 / 2$ defects should relate to one another as $4: 2: 1$. Thus, one would possibly expect the +2 disclination to go immediately into four $+1 / 2$ defects, but that does not happen.

There are two possible explanations for the mechanism of this cascade division. First, the rate of separation into two +1 disclinations may be greater than that into four $+1 / 2$ defects, so that it takes longer to form the $+1 / 2$ defect structure compared to the +1 structure. Secondly, the core energy of two +1 defects may be lower than the core energy of four $+1 / 2$ defects at the same distance from the centre. It is the second mechanism that we believe is the principal effect.

The calculated energy of two +1 defects or four $+1 / 2$ defects depends on the distance from the centre. Figure 5 shows the free 


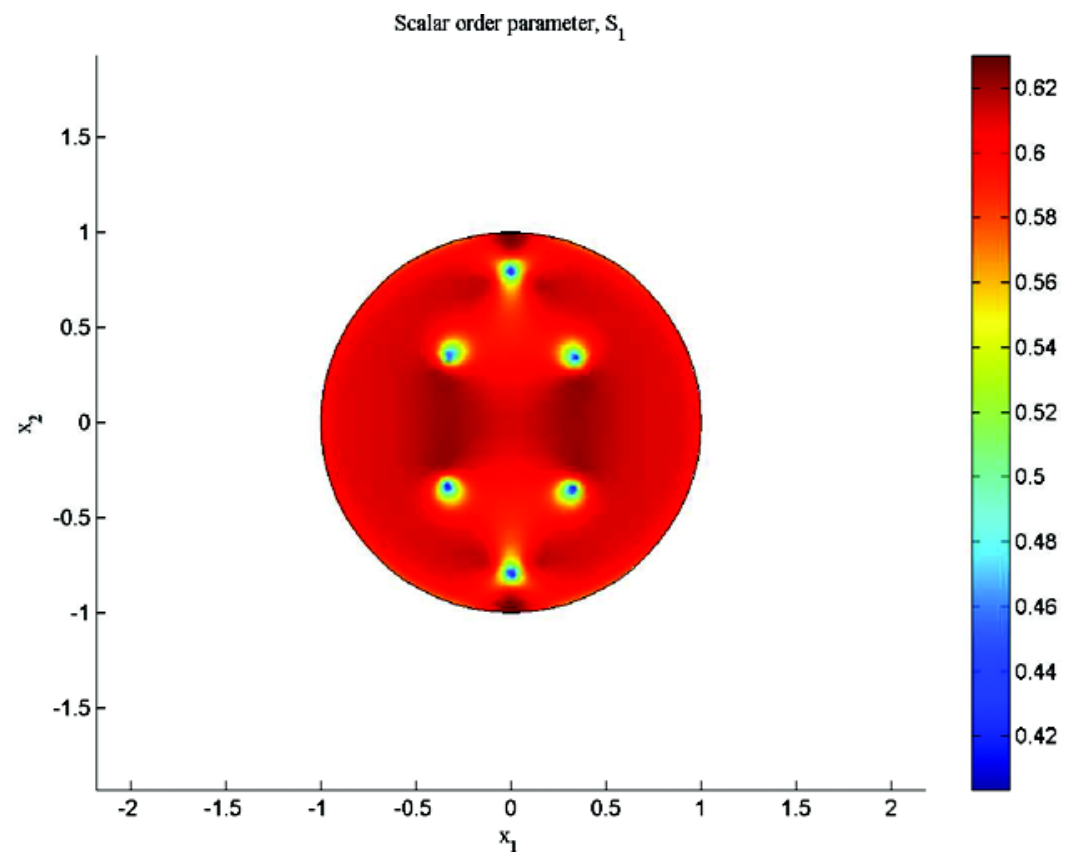

FIGURE 8 Surface plot for the scalar order parameter $S_{1}$. The two $-1 / 2$ and four $+1 / 2$ defect structure at $\bar{t}=0.022$ is shown here. $\bar{W}=8.0$.

energy $\mathscr{F}$ computed for three structures (by dropping the term with $F_{0}$ and taking $W=0 \mathrm{~N} / \mathrm{m}$ ): a +2 disclination in the centre, two +1 defects that have emerged in a very short time $(\bar{t}=0.001)$, and subsequently four $+1 / 2$ defects soon $(\bar{t}=0.0056)$ after the final division. The other set of energies shown are for two model director fields that are deliberately seeded in the domain at fixed points instead of the computed configuration. These have defect cores of radius 0.02 and the core boundary condition is set to $\theta=0, \psi=0$, $S_{1}=0.480, S_{2}=0.286$. These data are taken from the computed dynamics. One director field has four $+1 / 2$ defects each fixed symmetrically at the separation distance $\bar{d}_{1}=0.09$ from the centre (the same distance as for the two +1 defects in the computed dynamics) and the other has four $+1 / 2$ defects each fixed symmetrically at $\bar{d}_{1 / 2}=0.21$ from the centre (the same distance as for the four $+1 / 2$ defects in the computed dynamics). On the core boundary (and also as initial condition), the director field for one of the four $+1 / 2$ defects in the first model is given by $\tan (1 / 2) \varphi=\left(x_{2}-d_{1}\right) /\left(x_{1}+d_{1}\right)$ and similarly for 


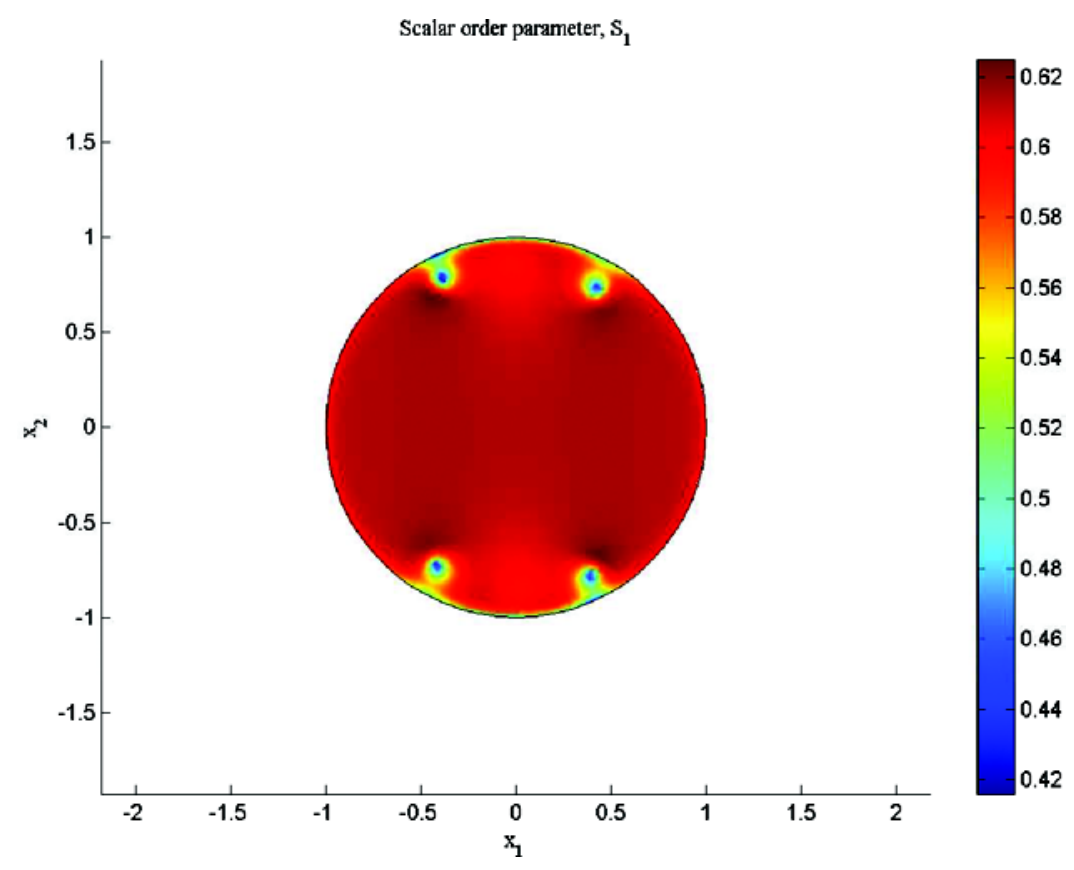

FIGURE 9 Surface plot for the scalar order parameter $S_{1}$. The four $+1 / 2$ defects have migrated to the bounding surface $(\bar{t}=0.075) . \bar{W}_{\mathrm{cr} 4}=2.9$.

the others and for the defects in the second model. The energies were calculated at times $\bar{t}=0.001$ and $\bar{t}=0.0056$ for the first and second model respectively. The data in Figure 5 show that when the separation distance is $d_{1}$ the computed two +1 defect state has a lower energy compared to the four $+1 / 2$ defects in the first model, but that the computed four $+1 / 2$ defect state has, as expected, almost the same energy as that of the four $+1 / 2$ defects in the second model at the distance $d_{1 / 2}$ from the centre. The final division itself actually occurs just before $\bar{t}=0.0056$ near where the lines cross in Figure 5. This qualitative comparison suggests that the cascade division is more energetically favourable and that the separation into four $+1 / 2$ defects is inevitable.

Another interesting observation is the relationship between $\mathbf{n}$ and m director fields in defect cores. Almost everywhere within the circular cross-section the nematic liquid crystal is uniaxial and $S_{2}=0$. However, the structure around the centre of defects arranges biaxially as $S_{1}$ and $S_{2}$ are different and non-zero. In the core of a planar +1 


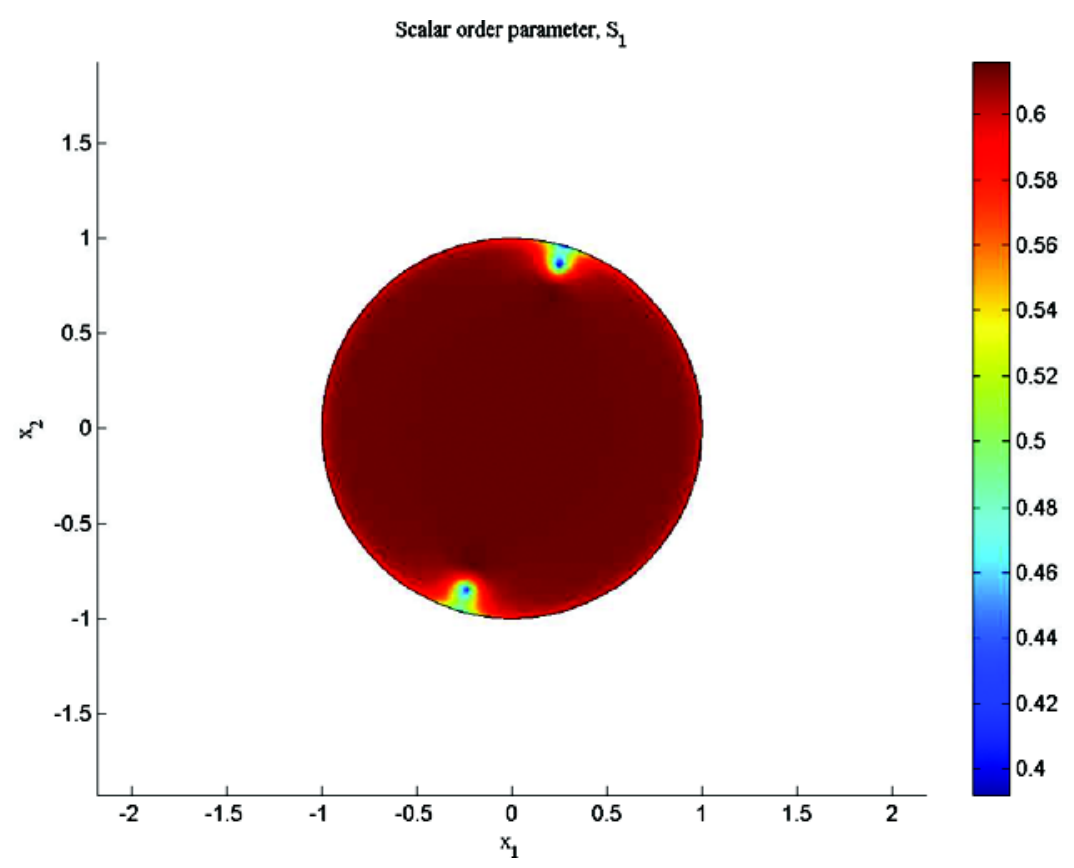

FIGURE 10 Surface plot for the scalar order parameter $S_{1}$. Two $+1 / 2$ defects have migrated to the bounding surface. $\bar{W}_{\mathrm{cr} 2}=5.367$.

defect, for instance, directors $\mathbf{n}$ and $\mathbf{m}$ may (usually do) form hedgehog (star) and vortex (circular) patterns, whereas in the core of a planar +2 defect directors $\mathbf{n}$ and $\mathbf{m}$ have the structures that can be obtained from each other simply by rotating a director field map by $\pi / 2$ angle. However, in our particular situation when the +2 disclination has just recently decayed into two planar +1 defects, both directors $\mathbf{n}$ and $\mathbf{m}$ form double vortex director fields (Figs. 6 and 7) that would match if one of them were rotated by an angle of $\pi / 2$.

\section{Anchoring Effects}

What happens to the four $+1 / 2$ defects after division depends on the boundary conditions. The dependence of specific director configurations within a liquid crystalline material on the angle at which liquid crystal molecules are anchored to the substrate was noticed [16] a long time ago. In our study, homeotropic boundary conditions with strong anchoring generate two $-1 / 2$ defects (Fig. 8) to counteract two of 
the four $+1 / 2$ disclinations in order to ensure the topological charge of +1 within the whole circular cross-section on aggregate. As defects with strengths that are of the same sign repel each other and those with different sign are attracted, the two $-1 / 2$ and two $+1 / 2$ defects annihilate one another and only two $+1 / 2$ defects will be left. However, that does not happen when the anchoring is sufficiently weak $\left(\bar{W} \leq \bar{W}_{\mathrm{cr} 4}, \bar{W}_{\mathrm{cr} 4}=2.9\right)$ and instead four $+1 / 2$ defects migrate towards the surface (Fig. 9) and disappear, although two very weak $+1 / 2$ defects still remain at opposite poles.

The final destination of the remaining two $+1 / 2$ defects in a final static pattern may be either their equilibrium positions (as shown in Fig. 2) at some distance from the boundary $\left(\bar{W}_{\mathrm{cr} 2}<\bar{W}\right)$ or right on the bounding surface $\left(\bar{W}_{\text {cr0 }}<\bar{W} \leq \bar{W}_{\text {cr2 }}, \bar{W}_{\text {cr2 }}=5.367\right.$, Fig. 10). With a particularly small anchoring strength $\left(\bar{W} \leq \bar{W}_{\text {cr0 }}, \bar{W}_{\text {cr0 }}=0.815\right)$, the defects escape from the boundary and a stable arrangement of directors oriented in one particular direction will emerge. Equilibrium positions of $+1 / 2$ defects are controlled by the anchoring strength.

\section{CONCLUSIONS}

The dynamics of liquid crystal ordering considered here within the circular cross-section of the cylinder are in contrast to the results in [10-14] for the escaped-radial director field of hyperbolic type. A cascade mechanism of defect division starting with the +2 disclination in the centre, then proceeding with two +1 defects, and finally reaching four $+1 / 2$ defect structure is discovered. The critical non-zero value of the anchoring strength when $+1 / 2$ defects escape from the picture is established. Although we appreciate the fact that it is presumably very difficult to create a +2 defect in a laboratory (as the dimensions are too small, time is too short and a +2 defect has to be seeded somehow), some experimental confirmation of these results is still desirable.

The tensor order parameter approach allows us to eliminate problems with the multivaluedness of the Euler angles and to avoid a singularity in the core of defects. Further numerical work on the system with a wider length scale suggests that the nematic director field might escape into the third dimension provided that the crosssection of the circular cylinder were sufficiently large.

\section{REFERENCES}

[1] Meyer, R. B. (1969). Phys. Rev. Lett., 22, 918.

[2] Dubois-Violette, E. \& Parodi, O. (1969). J. Phys. Colloq., C4-57. 
[3] Craighead, H. G., Cheng, J., \& Hackwood, S. (1982). Appl. Phys. Lett., 40, 22.

[4] Doane, J. W., Vaz, N. S., Wu, B. G., \& Žumer, S. (1986). Appl. Phys. Lett., 48, 269.

[5] Fergason, J. L. (1985). Soc. Inf. Disp. Dig., XVI, 68.

[6] Drzaic, P. S. (1986). J. Appl. Phys., 60, 2142.

[7] Crawford, G. P. \& Žumer, S. (Eds.) (1996). Liquid Crystals in Complex Geometries, Taylor \& Francis: London.

[8] Lehmann, O. (1907). Die Scheinbar lebenden Kristalle, Esslingen.

[9] Kléman, M. (1983). Points, Lines and Walls, John Wiley \& Sons.

[10] Cladis, P. E. \& Kléman, M. (1972). J. Phys., 40, 591.

[11] Williams, C. E., Pieranski, P., \& Cladis, P. E. (1972). Phys. Rev. Lett., 29, 90.

[12] Saupe, A. (1973). Mol. Cryst. Liq. Cryst., 21, 211.

[13] Meyer, R. B. (1973). Phyl. Mag., 27, 405.

[14] Bunning, J. D. \& Lydon, J. E. (1996). Liq. Cryst., 20, 381.

[15] Lee, H. \& Labes, M. M. (1982). Mol. Cryst. Liq. Cryst., 82(Letters), 199.

[16] Lehmann, O. (1904). Flüssige Kristalle, Verlag von Wilhelm Engelmann: Leipzig.

[17] de Gennes, P. G. \& Prost, J. (1993). The Physics of Liquid Crystals, Claredon Press: Oxford.

[18] Virga, E. G. (1994). Variational Theories for Liquid Crystals, Chapman \& Hall.

[19] Strutt, J. W. (Lord Rayleigh) (1873). Proc. Lond. Math. Soc., 4, 357.

[20] Sonnet, A. M. \& Virga, E. G. (2001). Phys. Rev. E, 64, 031705.

[21] Landau, L. D. (1965). Collected Papers, Ter Haar, D. (Ed.), Gordon and Breach: New York, 193.

[22] Coles, H. J. (1981). Mol. Cryst. Liq. Cryst., 49(Letters), 67.

[23] Mori, H., Gartland, E. C., Kelly, J. R., \& Bos, P. J. (1999). Japan J. Appl. Phys., 38, 135.

[24] Bunning, J. D., Faber, T. E., \& Sherrell, P. L. (1981). J. Phys., 42, 1175.

[25] Lydon, J. E. (2003). Personal communication.

[26] Karat, P. P. \& Madhusudana, N. V. (1976). Mol. Cryst. Liq. Cryst., 36, 51.

[27] Karat, P. P. \& Madhusudana, N. V. (1977). Mol. Cryst. Liq. Cryst., 40, 239. 